\title{
Immunological response to re-infections with clones of the Colombian strain of Trypanosoma cruzi with different degrees of virulence: influence on pathological features during chronic infection in mice
}

\author{
Marcos Lazaro da Silva Guerreiro, Isa Rita Brito Morais, Sonia Gumes Andrade/+ \\ Laboratório de Chagas Experimental, Autoimunidade e Imunologia Celular, Centro de Pesquisas Gonçalo Moniz-Fiocruz, Salvador, BA, Brasil
}

Re-infections with Trypanosoma cruzi are an aggravating factor for Chagas disease morbidity. The Colombian strain of T. cruzi represents multiclonal populations formed by clonally propagating organisms with different tropisms and degrees of virulence. In the present study, the influence of successive inoculations with clones of the Colombian strain, exhibiting different degrees of virulence, on chronic myocarditis and the humoral and cellular immune responses (Col-Cl high virulence, Col-C8 medium virulence and Col-C5 low virulence) were demonstrated. Mice from three groups with a single infection were evaluated during the acute (14th-30th day) and chronic phases for 175 days. An immunofluorescence assay, ELISA and delayed type hypersensitivity (DTH) cutaneous test were also performed. Mice with a triple infection were studied on the 115th-175th days following first inoculation. The levels of IgM and IgG $G_{2 a}$ were higher in the animals with a triple infection. DTH showed a higher intensity in the inflammatory infiltrate based on the morphometric analysis during a $48 \mathrm{~h}$ period of the triple infection and at $24 \mathrm{~h}$ with a single infection. The histopathology of the heart demonstrated significant exacerbation of cardiac inflammatory lesions confirmed by the morphometric test. The humoral responses indicate a reaction to the triple infection, even with clones of the same strain.

Key words: T. cruzi - re-infections - clones - myocarditis - DTH - serology

The possibility that individuals living in endemic areas of Chagas disease are subjected to repeated inoculations with Trypanosoma cruzi is a factor influencing Chagas disease morbidity (Macedo 1976, Dias et al. 2002, Bustamante et al. 2003, Andrade et al. 2006). According to Coura (2013), in an endemic area of Chagas disease, re-infections are common. Several types of relationships with infected men or wild animals are possible contamination sources, either "as an enzooty of wild animals and vectors or as an anthropozoonosis". The multiclonality of T. cruzi strains allows for the isolation of clones with different degrees of virulence, even from strains isolated from the same endemic areas and presenting with the same biological, isoenzymic or genetic profiles, as previously shown (Campos \& Andrade 1996) for the 21SF strain from São Felipe (state of Bahia, Brazil) and with the Colombian strain (Camandaroba et al. 2001). In a large clinical-epidemiological study from the endemic area of São Felipe, Macedo (1976) compared patients from localities where the vector had been eliminated with those from areas where the insect remained in the houses. They detected differences in the clinical manifestations and morbidity of Chagas disease. It is possible that the exposure of patients to multiple contacts with the vector determines $T$. cruzi infections with multiple dif-

doi: 10.1590/0074-02760140286

+ Corresponding author: sgandrade@bahia.fiocruz.br

Received 7 August 2014

Accepted 12 February 2015 ferent strains of the parasite or with different clones of the same parasite strain. In previous experimental studies (Andrade et al. 2006), triple infections with strains of different biodemes were associated with aggravation of the histopathological lesions. The persistence of the three strains in these triply infected animals was confirmed. This is important because the three strains differ in their virulence and pathogenicity and their persistence in the triply infected mice may be responsible for the peculiar severity of the cardiac lesions observed.

It has been hypothesised that the predominance of the most virulent and, consequently, most pathogenic clones is related to the immunological responses and the degree of myocarditis in these experimental animal infections. To investigate this possibility, clones with different degrees of virulence that were isolated from the Colombian strain were successively inoculated into mice and the infection, mortality rates and histopathological lesions were evaluated. In addition, the humoral and cellular immunological responses were assessed. The importance of this hypothesis is related to the control measures of infection transmission and the decreased morbidity of Chagas disease.

\section{MATERIALS AND METHODS}

Strain of T. cruzi - The Colombian strain of T. cruzi, isolated by Federici et al. (1964), was used in the present study. This strain is classified as biodeme type III (Andrade 1974), zymodeme I (Andrade et al. 1983, Andrade \& Magalhães 1997) and T. cruzi I (Anonymus 1999). The clones used for this study were isolated by Camandaroba et al. (2001).

Cloning of the strain - To obtain a single parasite, citrated blood collected from mice infected with the parental strain on the 30th day of infection was centrifuged at $900 \mathrm{~g}$ and the parasites in the plasma were counted in 
a Neubauer chamber after dilution in phosphate buffered saline (PBS), $\mathrm{pH}$ 7.2. A volume of $1 \mathrm{~mL}$ was distributed into multiwell microtitre culture plates and examined with an inverted microscope. By micromanipulation, a single trypomastigote form was isolated and intraperitoneally inoculated into a suckling mouse (8-days-old). From 10-30 days after the inoculation, the peripheral blood was examined for the presence of trypanosomes, which were then defined as clones and classified as high virulence (Col-C1), medium virulence (Col-C8) and low virulence (Col-C5). The clones were maintained in cryopreservation in liquid nitrogen at $-196^{\circ} \mathrm{C}$. For inoculation, the clones were thawed at $37^{\circ} \mathrm{C}$ and immediately inoculated into Swiss mice (weighing 10-12 g) to obtain the inoculum for the experimental groups.

Experimental groups - In the present study, 160 Swiss mice of both sexes weighing 18-25 g were intraperitoneally infected with $5 \times 10^{4}$ trypomastigote blood forms as follows: (i) group I - triple infection - 80 mice were infected successively with the three clones at intervals of 50 days [Col-C5 (low virulence); Col-C8 (medium virulence); Col-C1 (high virulence)] and (ii) group II - single infection (controls divided in 2 subgroups) - 40 mice were infected with clone $\mathrm{Col}-\mathrm{C} 1$ and 40 mice were infected with clone Col-C8.

Follow up of the experimental groups - Parasitaemia was evaluated on the seventh-50th day after infection in five mice by microscopic examination of $5 \mu \mathrm{L}$ of fresh tail blood. The blood was mounted between the glass and a cover-slip and the parasites were counted in $50 \mathrm{mi}-$ croscopic fields at 400X.

Mortality - The mortality was daily evaluated and recorded as the percentage of survivors during the experiments.

Histopathological study - The mice from the groups were euthanised by exsanguination after anaesthesia with ketamine/xylazine for the histopathological study. The blood was collected from each mouse for haemoculture. Complete autopsies were performed and several organs were fixed in $10 \%$ buffered formalin. The tissues were embedded in paraffin and $5 \mu \mathrm{m}$ sections were stained with haematoxylin and eosin (H\&E) for histopathological study.

Single infection - The animals with a single infection with each clone were sacrificed during the acute phase (14th, 20th, 25th and 30th day post-infection) and chronic phase (150th day post-infection).

Triple infection - In the group of triply infected mice, the mice were sacrificed after each infection at the same time points as the animals with the single infection (14th, 20th, 25th, 30th and 175th day after the 1st infection). Sections of the heart were fixed in formaldehyde/Millonig solution and embedded in paraffin. Sections of $5 \mu \mathrm{m}$ were stained with H\&E or Picrosirius for collagen deposits.

Semiquantitative evaluation of the inflammatory infiltrate - A semiquantitative analysis of the inflammatory infiltrates was performed and recorded as,+++ and +++ according to the severity of the lesions as follows: + , mild focal or diffuse mononuclear infiltration, ++ , moderate diffuse and focal infiltrates, and +++, intense focal and diffuse inflammatory infiltration and necrosis of myocells.

Morphometric evaluation of the inflammatory infiltration - Evaluation of the number of inflammatory cells was performed by microscopic counting in five $12 \mathrm{~mm}^{2}$ fields, with an ocular 10X and an objective 40X, with an area of $60 \mathrm{~mm}^{2}$. The images were captured and evaluated using the program FACSort flow cytometer (Immunocytometry, Becton Dickinson, USA) and a FASCsAria cell sorter (BD Biosciences). All data were analysed using FlowJo software (USA). The results were evaluated with the non-parametric Mann-Whitney $U$ test with a significance of $\mathrm{p}<0.05$.

Indirect immunofluorescence serological test - This assay was performed according to Camargo (1966). The reaction was performed with different serum concentrations ranging from 1:10-1:1280, in multiwell slides using as antigens culture forms of $T$. cruzi and $10 \mu \mathrm{L}$ of antimouse IgG fluorescein conjugated (Sigma) as specific antibodies. The slides were analysed on a Zeiss epifluorescence microscope of with halogen lamp.

Immunoglobulin dosage evaluation by ELISA - The wells were coated with $T$. cruzi antigen, prepared by alkaline extraction of Colombian strain and harvested at the exponential growth stage in Warren medium (Warren 1960). The binding of antibodies was detected using peroxidase-labelled anti-mouse $\operatorname{IgM}, \operatorname{IgG} 1$ and $\operatorname{IgG} 2 \mathrm{a}$ isotype-horseradish peroxide conjugates (Bethyl Laboratories, USA) and the absorbances were read at 450 $\mathrm{nm}$. To optimise the serum dilutions, the samples were titrated from 1:20-1:1,240 and the assays were performed with four samples, each presenting with high, medium and low antibody reactivities, in parallel with the four samples from noninfected mice. A good discrimination was observed at 1:100 and this dilution was used for all further assays. The cut-off value is the mean \pm 2 standard deviations of the negative sample $(n=20)$.

Skin tests - To evaluate the cellular immune responses, a delayed type hypersensitivity (DTH) skin test was performed using the antigen culture forms of $T$. cruzi with $90 \%$ epimastigotes. The cells were washed in PBS (pH 7.2) with centrifugation. The pellet containing the culture forms was frozen and thawed several times in liquid nitrogen. The antigenic extract was then filtered in a Millipore $0.22 \mu \mathrm{m}$ filter. The protein concentration was determined using the bicinchoninic acid assay (BCA) with a BCA Protein Assay Kit (Pierce; catal. 2161297A). The protein concentration for the sample used was adjusted to $2 \mathrm{mg} / \mathrm{mL}$.

The skin test was performed in 18 mice from each experimental group at three time points, $24 \mathrm{~h}, 48 \mathrm{~h}$ and $72 \mathrm{~h}$, after antigen administration in a dose of $25 \mu \mathrm{L}(50$ $\mu \mathrm{g}$ of protein) intradermally administered in the right hind-footpad. The same volume of PBS was injected into the left hind-footpad and served as a control. The footpad thickness was measured with a digital calliper (Fisherbrand Digital Callipers, Traceable, Fisher Scientific) at $24 \mathrm{~h}, 48 \mathrm{~h}$ and $72 \mathrm{~h}$ after the antigen injection. 


\section{RESULTS}

Parasitaemia (Fig. 1A) - In the animals with a single infection with each clone, the parasitaemia became positive on the eighth day of infection, with peaks from the 19th-20th day. In the group of mice with the triple infection, the parasitaemia remained negative after the second and third infections.

Cumulative mortality (Fig. 1B) - Group Col-C1 presented a cumulative mortality of $100 \%$ until the 30th day post-infection. The other groups presented cumulative mortalities of $23 \%$ (Col-C5), $50 \%(\mathrm{Col} \mathrm{C} 8)$ and $60 \%$ (triple infected mice).

Indirect immunofluorescence assay - The anti-T. cruzi serological reaction showed, for the groups with single infection (Table I) in the acute phase, titres that varied from 1:10-1:180 and, for infection in the chronic phase (150 days), titres that varied from 1:10-1: 640 (Table I). In the triply infected mice, the titres varied from 1:10-1:2,560 (115th-150th days post the third infection) (Table II).

ELISA (Fig. 2A-C) - The levels of IgG2a and IgM were higher in the mice with successive re-infections than in those with a single infection. The IgG1 isotype was significantly lower in the mice with the triple infection than in the group with a single infection and the controls.
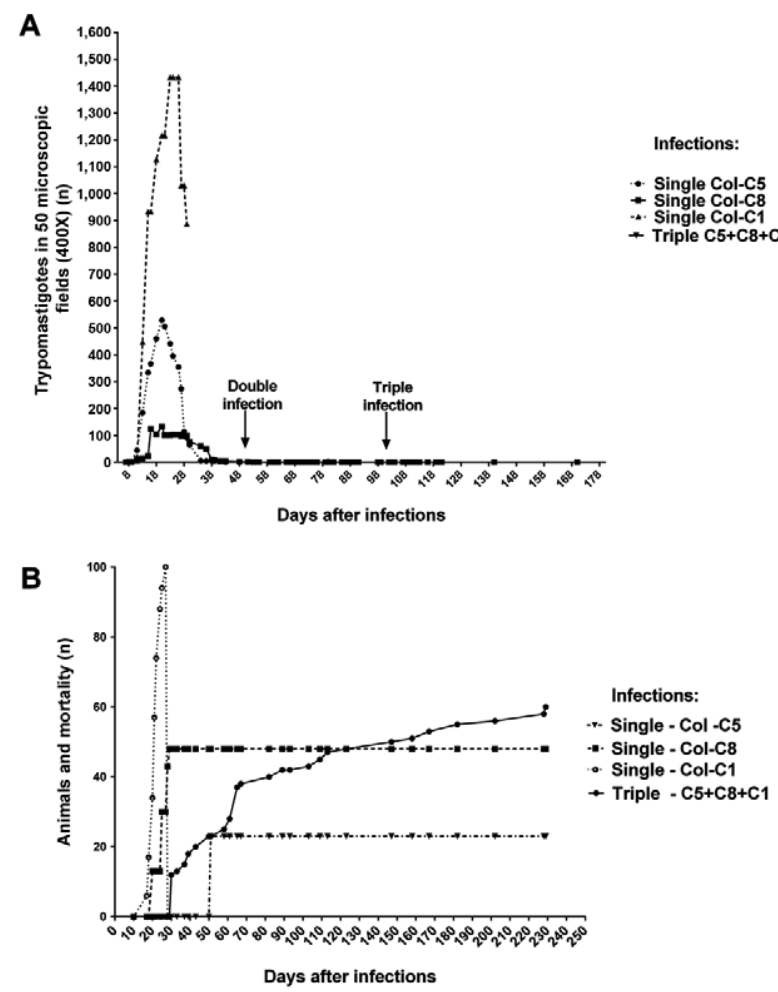

Fig. 1A: parasitaemic profiles in mice infected with the clones Col$\mathrm{C} 1, \mathrm{Col}-\mathrm{C} 5, \mathrm{Col}-\mathrm{C} 8$ and triple infected. In the several groups the parasitaemic peak is around the 20th day of infection; B: cumulative mortality in the different experimental groups inoculated with clones of different degrees of virulence.
Histopathology of the heart - The experimental groups of mice with a single infection with each clone isolated from the Colombian strain, as well as the group with the triple infection, were analysed in the acute and chronic phases of infection. The intensity of the inflammatory infiltrates, the necrotic lesions of cardiac myocells and the presence of intracellular parasites were assessed.

Group of single infection - Acute phase (Fig. 3A, B), mice infected with the clone $\mathrm{C} 1$ (high virulence) 14th30th day post-infection: the lesions of the myocardium varied from mild to moderate with diffuse and focal mononuclear inflammatory infiltrates, necrosis of myocytes and moderate parasitism. In this group, the chronic phase was not evaluated because all the mice died by the 30th day post-infection.

Group of single infection - (i) Acute phase (Fig. 3C, D): mice infected with clones Col-C5 and Col-C8 (low and medium virulence). The histopathological study showed mononuclear, moderate, diffuse or focal infiltrates, with destruction of the cardiac myocells and moderate parasitism; (ii) chronic phase (150 days) of single infection (Fig. 3E, F): for infection with clones Col-C5 and $\mathrm{Col}-\mathrm{C} 8$, the inflammatory lesions of the myocardium varied from mild to moderate based on the morphometric analysis of the inflammatory cell infiltration

\section{TABLE I}

Titres of specific antibodies in mice in the acute phase of infection with clones $\mathrm{Col}-\mathrm{C} 1, \mathrm{Col}-\mathrm{C} 5$, Col-C8, respectively, and triple infected

\begin{tabular}{lcccc}
\hline & & & \multicolumn{2}{c}{ Antibody titres } \\
\cline { 4 - 5 } Identification & Inocula & $(\mathrm{n})$ & Lower & Higher \\
\hline Col-C1 & $5 \times 10^{4}$ & 12 & $1 / 10$ & $1 / 180$ \\
Col-C5 & $5 \times 10^{4}$ & 12 & $1 / 10$ & $1 / 180$ \\
Col-C8 & $5 \times 10^{4}$ & 12 & $1 / 10$ & $1 / 180$ \\
Col-triple & $5 \times 10^{4}$ & 12 & $1 / 10$ & $1 / 1,280$ \\
\hline Total & & 48 & \multicolumn{2}{c}{ Antibody level } \\
\hline
\end{tabular}

\section{TABLE II}

Titres of specific antibodies in mice in the chronic phase of infection with clones Col-C1, Col-C5, Col-C8, respectively, and triple infected

\begin{tabular}{lcccc}
\hline & & \multicolumn{2}{c}{ Mice } & \multicolumn{2}{c}{ Antibody titres } \\
\cline { 4 - 5 } Identification & Inocula & $(\mathrm{n})$ & Lower & Higher \\
\hline Col-C5 & $5 \times 10^{4}$ & 5 & $1 / 10$ & $1 / 320$ \\
Col-C8 & $5 \times 10^{4}$ & 5 & $1 / 10$ & $1 / 640$ \\
Col-triple & $5 \times 10^{4}$ & 5 & $1 / 10$ & $1 / 2560$ \\
\hline Total & & 15 & \multicolumn{2}{c}{ Antibody level } \\
\hline
\end{tabular}



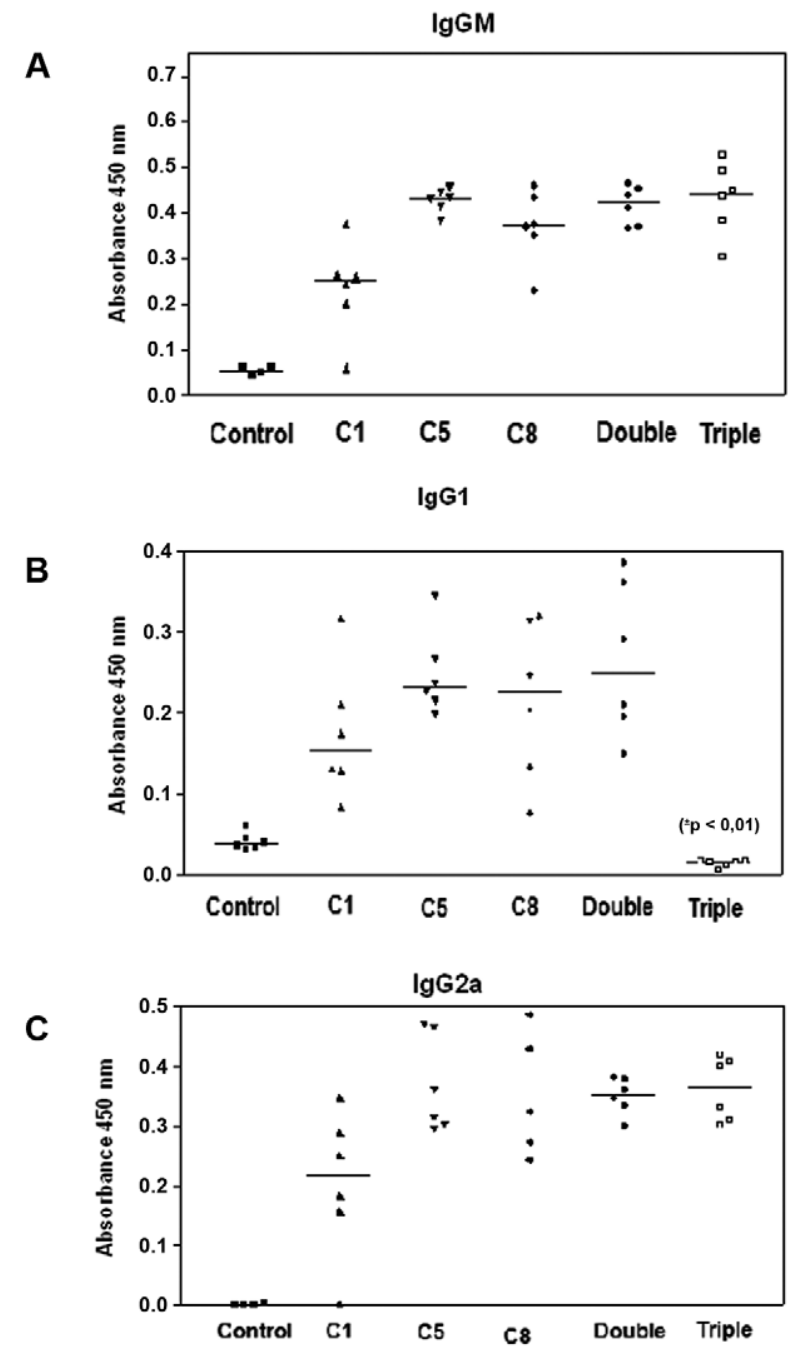

Fig. 2: serological levels of immunoglobulins in the acute and chronic phases of single and triple infection in mice infected with the clones Col-C1 (high virulence), Col-C8 (medium virulence) and Col-C5 (low virulence). A, C: higher levels of IgM and IgG2a were detected in the mice with triple infection as compared with those with single infection; B: the IgG1 isotype showed significantly lower levels ( $p$ $<0.01$ ) in the mice submitted to triple infection, as compared with those with single infection and controls.

in the hearts of mice with a single infection compared to the mice with a triple infection $(\mathrm{p}<0.0001)$ (Fig. 4B). In the chronic phase, the presence of interstitial fibrosis was observed and focal deposits of collagen were observed that were positive for picrosirius (Fig. 3G, H).

Group of triple infection (ColC5 + ColC $8+$ ColCl) - After the third infection with the high virulence clone Col-C1, histopathological evaluation was performed on the 14th, 20th and 30th day post-infection. At the 14th day, focal necrosis of the myocardium was present. At the 20th day post-infection, intense and diffuse inflammatory lesions with moderate parasitism of the heart were observed (Fig. 5A, B). Lesions of the heart increased at the 30th day, with intense inflammatory infiltrations and significant parasitism of the myocardium
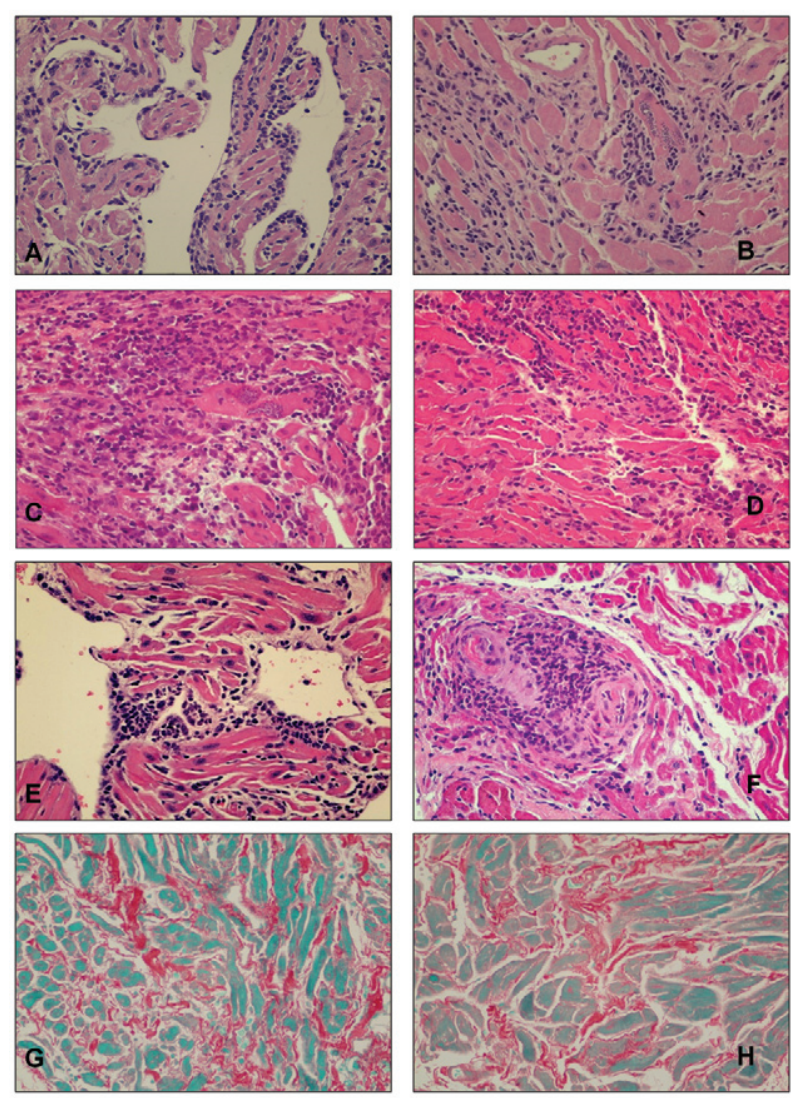

Fig. 3A, B: sections of mouse atrial myocardium in the acute phase of single infection with clone Col-C1 (high virulence), showing intense subendocardic mononuclear cells infiltration; C: sections of the myocardium showing mononuclear, moderate, diffuse or focal infiltrates with destruction of cardiac myocell and moderate parasitism; D: section of the ventricle with moderate and diffuse inflammatory infiltrations and the presence of amastigotes of Trypanosoma cruzi into the heart myocells; F: sections of the heart in chronically infected mouse (single infection) clone Col-C5 (low virulence) showing focal mononuclear infiltrates in the ventricular myocardium; E: heart mouse with single infection with clone Col-C8 (medium virulence) showing an arteriolar involvement with intense focal periarteriolar infiltrate and necrosis of arteriolar media; G, H: sections of the myocardium of chronically infected mouse with clones Col-C5 and Col-C8 (respectively) showing interstitial collagen deposits, stained positive with picrosirius red (A-F: 400X).

(Fig. 5C, D). Inflammatory infiltration of the heart was evaluated by morphometric analysis and showed a significant increase in the number of inflammatory cells in the triple infection compared to the single infection $(\mathrm{p}<0.0001)$ (Fig. 4B). Interstitial fibrosis was present and positive for picrosirius staining (Fig. 5E, F).

Histopathological evaluation of DTH - (i) Skin test in mice with single infection: mice with a single chronic infection at $24 \mathrm{~h}$ and $48 \mathrm{~h}$ after antigen injection showed focal and mild diffuse polymorphonuclear neutrophil infiltrates and diffuse mononuclear cell infiltration in the dermis and subdermic conjunctive tissue involving small vessels (venules and arterioles) $(\mathrm{p}<0.05)$ (Fig. 6A); (ii) skin test in mice with triple infection: the inflammatory response was more robust within $24 \mathrm{~h}$ with increased myositis and poly- 
A

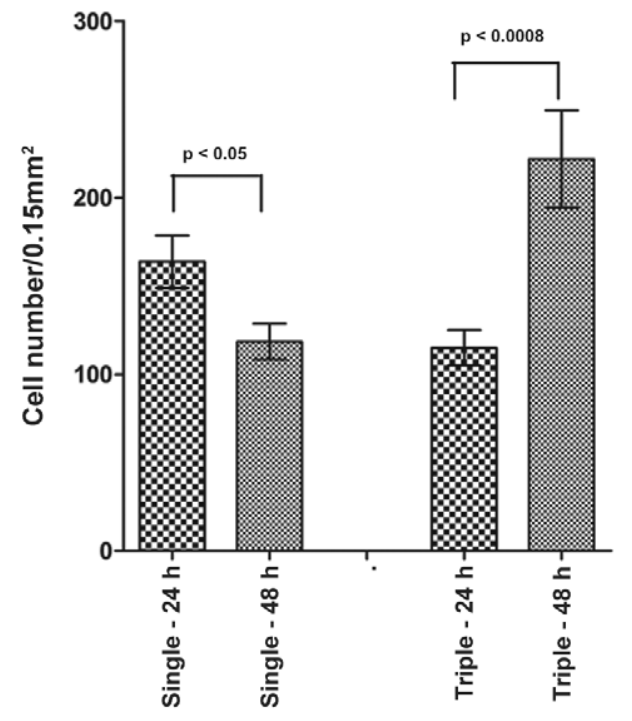

B

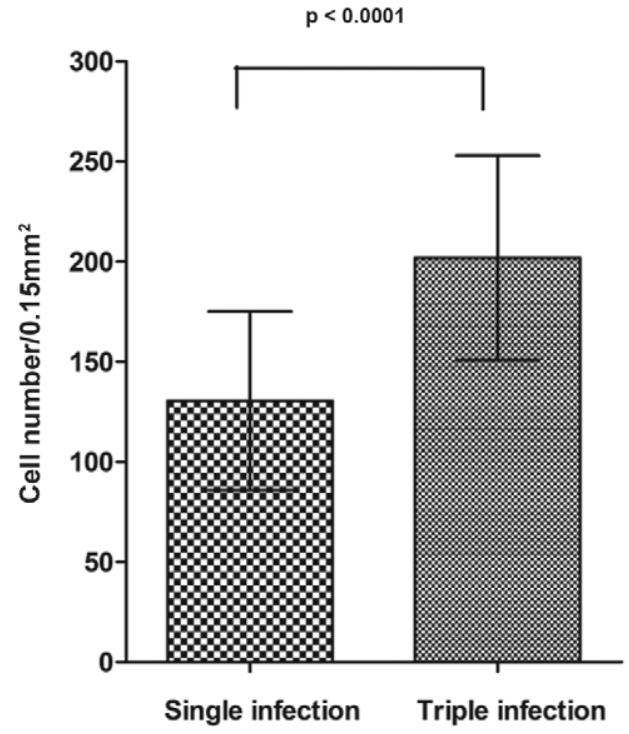

Fig. 4A: cutaneous test morphometric evaluation of the inflammatory infiltrates in the dermis of the mice with single and triple infection with $24 \mathrm{~h}$ and $48 \mathrm{~h}$. Significant difference between $24-48 \mathrm{~h}(\mathrm{p}<0.0008)$ was also detected in the nonparametric test Mann-Whitney $U$; B: morphometric evaluation of the inflammatory infiltrates in the myocardium. Significant differences $(p<0.0001)$ were seen between the mice with single infection with each clone and those with triple infections.

morphonuclear cell infiltration involving the vessels and nervous fibres. These lesions extended to the subdermal tissue with diffuse mononuclear cell infiltration and the involvement of vessels and cutaneous annexes. After $48 \mathrm{~h}$, the lesions were similar but less intense than those observed $24 \mathrm{~h}$ post antigen injection $(\mathrm{p}<0.0008)$ (Fig. 6B-D).

Morphometric evaluation of the inflammatory infiltrates - Cutaneous test: the morphometric evaluation of the inflammatory infiltrates of the dermis in mice with a single infection was significantly different from that at $24-48 \mathrm{~h}(\mathrm{p}<0.05)$. In the group with the triple infection,

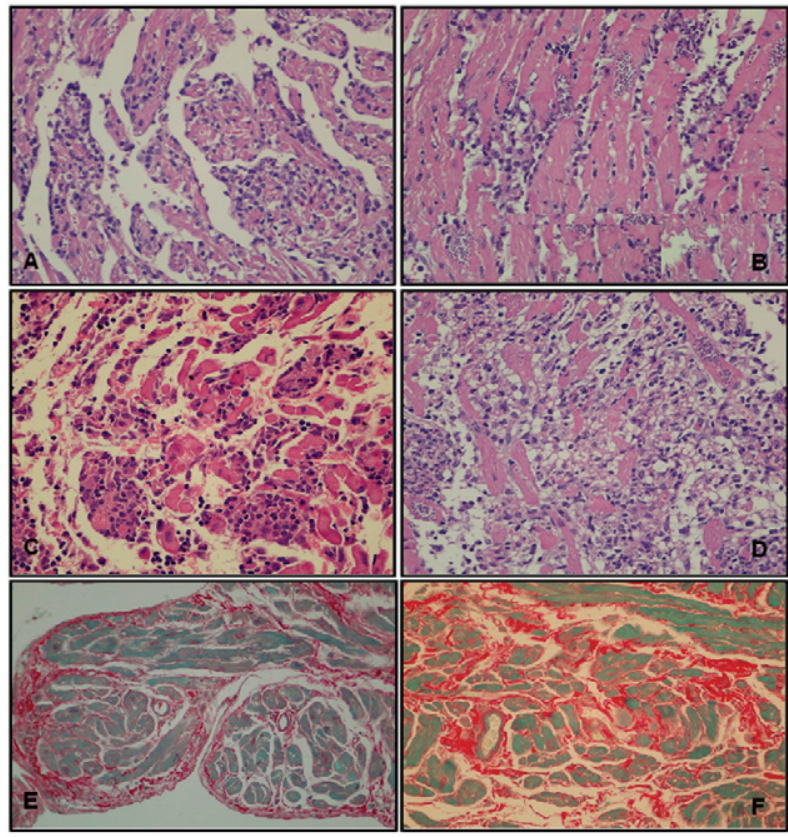

Fig. 5: sections of the myocardium in mice with triple infection 175 days after the first inoculation. A: atrial myocardium with focal areas of necrosis moderate and diffuse inflammatory infiltrations and scarce parasitism of the myocardium; B: section of the ventricle muscle showing moderate mononuclear cells infiltration and presence of intracellular amastigotes of Trypanosoma cruzi parasitism; C, D: section of the heart of mouse with extensive area of necrosis of the myocardium with destruction of cardiac myocells and substitution by inflammatory mononuclear infiltrate and moderate parasitism of the heart; E, F: sections of the heart of triple infected mice; E: atrial myocardium with interstitial fibrosis identified by positive picrosirius red staining (400X); F: ventricular myocardium showing interstitial fibrosis identified with the picrosirius red staining (400X).

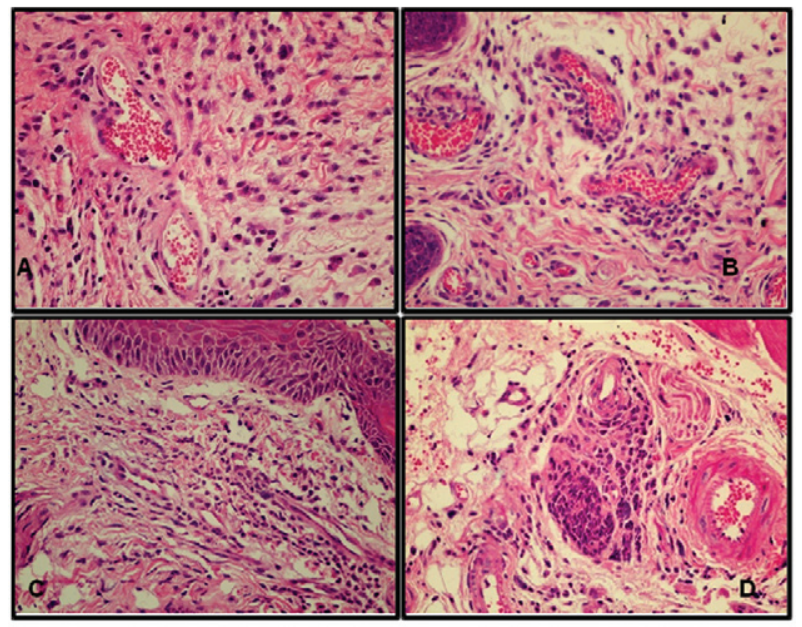

Fig. 6: delayed type hypersensitivity skin test in mice with single infection $(24 \mathrm{~h})$. A: focal, mild, diffuse polymorphonuclear cells infiltration around venules and arterioles and diffuse mononuclear infiltration; B-D: triple infection, lesions after $48 \mathrm{~h}$ are represented by diffuse mononuclear infiltration in the dermis and polymorphonuclear cells involving the vessels (venules and arterioles) and nervous fibres (400X). 
a significant difference between 24-48 h $(\mathrm{p}<0.0008)$ was also detected. For all groups, the Mann-Whitney $U$ nonparametric test was used (Fig. 4A); myocardium: significant differences were observed between the mice with a single infection with each clone and those with triple infections (Fig. 4B) with a higher number of inflammatory cells in the triply infected mice $(p<0.0001)$.

\section{DISCUSSION}

After the decrease on Chagas disease transmission via vector control in Latin America, there has been a reduction of morbidity due to Chagas disease (Dias et al. 2002). However, epidemiological studies revealed the presence of several outbreaks of Chagas disease in different geographical areas, generally resulting from oral transmission (Camandaroba et al. 2002, Andrade et al. 2011). This confirms the occurrence of re-infections due to different strains and clones of $T$. cruzi in several endemic areas (Macedo 1976). The results of this study demonstrate the influence of re-infections by clones isolated from the Colombian strain of T. cruzi (biodeme type III and genotype T. cruzi I) (Anonymus 1999) with different degrees of virulence on heart lesions and, consequently, its influence on chronic Chagas disease myocardiopathy. According to previous studies, (Andrade et al. 2006), successive re-infections with different strains of $T$. cruzi are responsible for the aggravation of myocarditis in infected mice. When studying the influence of the Colombian strain on chronic chagasic myocarditis using two different mouse strains, Pereira et al. (2014) showed the presence of high levels of tumour necrosis factor and nitric oxide in the serum directly associated with intense diffuse and focal inflammatory infiltration, which are consistent with our findings. A similar effect on the intensity of the inflammatory infiltrate was previously observed in dogs during the indeterminate phase of infection (Andrade et al. 1987) and in mice subjected to treatment with cyclophosphamide at low doses, likely due to the influence of this drug on the regulatory mechanism of DTH (Thé et al. 2013). The response to low doses of cyclophosphamide are attributed to the suppression of the humoral and cellular responses and the result of the selective destruction of suppressor T lymphocytes, their precursor cells or other elements in the host immune suppressor network (Andrade et al. 1987, Murata et al. 2004, Lutsiak et al. 2005, Thé et al. 2013). These cells are identified as regulatory T-cells (Tregs), which express $\mathrm{CD} 4{ }^{+} \mathrm{CD} 25^{+}$markers and are involved in the regulation or suppression of DTH (Shevach et al. 2001). Apparently, successive re-infections inhibit the suppressor mechanisms of Treg cells and intensify the inflammatory infiltrate in the myocardium.

In mice with a triple infection, the correlation between the humoral response and the intensity of the fibrotic-inflammatory lesions of the myocardium and the expression of the DTH was evaluated with the skin test using T. cruzi antigens. The pathogenesis of the inflammatory response in the chronic myocardiopathy of Chagas disease is related to several factors, such as the strain and clones of the parasite and its virulence, the immune response of the host and the regulatory mechanisms that control the cel- lular responses to infection. The virulence of each clone influences the mortality rates, which are lower with the low virulence clone ColC-5 (23\%) and 100\% during infection with the high virulence clone ColC-1. The groups of mice with successive inoculations failed to develop an acute phase of $T$. cruzi infection and did not show a positive parasitaemia after re-inoculations. In the present study, during the chronic phase of triple infection, severe myocarditis with high parasitism of the cardiac myocells was present. The single infection with clones of different degrees of virulence did not influence the serological response of the infected mice. However, three successive inoculations at 50 day intervals resulted in higher antibody titres after the second infection, which was likely due to the stimulation of immunological memory. In previous studies (Andrade et al. 1985) with inbred mice of different isogenic strains, T. cruzi infection resulted in variations of the serum immunoglobulins independently of the parasite strain, with an early decrease followed by a significant increase during infection. The IgM levels were consistently high, primarily after the 20th day after re-infection. The immunosuppression during the early phase of infection was likely due to the polyclonal activation of spleen lymphocytes, such as that which occurs during the initial phase of infection, with low levels of specific immunoglobulins (Andrade et al. 1985). The IgG2a levels showed an increase from the 20th day postinfection in several groups and were higher in the group with the triple infection, with increased parasitism and tissue lesions. The IgG1 isotype showed a significant reduction in the group with the triple infection. Different clonal isotypes of $T$. cruzi influence the expression of the different classes of immunoglobulins (dos Santos et al. 2009). It is important to note the influence of the DTH on the response of the mice to the multiple infections. In the present study, the mice in the chronic phase of the infection, either singly or triply infected, were subjected to the DTH test by the intradermal injection with the parasite antigens. Evaluation of the inflammatory response showed the presence of mononuclear cells and polymorphonuclear neutrophil infiltrations, which were increased $24 \mathrm{~h}$ and $48 \mathrm{~h}$ after the antigen injections. Morphometric evaluation of the inflammatory infiltrates in the myocardium using the nonparametric Mann-Whitney $U$ test revealed significant differences between the triple and single infections and were more intense in the $48 \mathrm{~h}$ post-injection during the chronic phase in triple infection and at $24 \mathrm{~h}$ in the mice with a single infection.

The results of this investigation confirm the importance and influence of successive re-infections with $T$. cruzi as a factor that aggravates the myocardiopathy of Chagas disease.

\section{REFERENCES}

Andrade SG 1974. Caracterização de cepas do Trypanosoma cruzi isoladas no Recôncavo Baiano. Rev Patol Trop 3: 121-165.

Andrade SG, Campos RF, Sobral KSC, Magalhães JB, Guedes RSP, Guerreiro MLS 2006. Reinfections with different strain of Trypanosoma cruzi as a factor of aggravation of myocarditis and myositis in mice. Rev Soc Bras Med Trop 39: 1-8. 
Andrade SG, Campos RF, Steindel M, Guerreiro ML, Magalhães JB, de Almeida MC, Reis JN, Santos VC, Valadares HMS, dos Reis MG, Macedo AM 2011. Biological, biochemical and molecular features of Trypanosoma cruzi strains isolated from patients infected through oral transmission during a 2005 outbreak in the state of Santa Catarina, Brazil: its correspondence with the new T. cruzi Taxonomy Consensus (2009). Mem Inst Oswaldo Cruz 106: 948-956.

Andrade SG, Magalhães JB 1997. Biodemes and zymodemes of Trypanosoma cruzi strains: correlations with clinical data and experimental pathology. Rev Soc Bras Med Trop 30: 27-35.

Andrade V, Barral-Netto M, Andrade SG, Magalhães JB 1985. Aspectos imunológicos da infecção de seis linhagens isogênicas de camundongos por três diferentes cepas do Trypanosoma cruzi. Mem Inst Oswaldo Cruz 80: 203-211.

Andrade V, Brodskyn C, Andrade SG 1983. Correlation between isoenzyme patterns and biological behavior of different strains of Trypanosoma cruzi. Trans R Soc Trop Med Hyg 77: 796-799.

Andrade ZA, Andrade SG, Sadigursky M 1987. Enhancement of chronic Trypanosoma cruzi myocarditis in dogs treated with low doses of cyclophosphamide. Am J Pathol 127: 467-473.

Anonymus 1999. Recomendations from a satellite meeting. Mem Inst Oswaldo Cruz 94 (Suppl. I): 429-432.

Bustamante JM, Rivarcia HW, Fernandez AR, Enders JE, Fretes R, Palma JÁ, Paglini-Oliva PA 2003. Indeterminate Chagas disease: Trypanosoma cruzi strain and re-infection are factors involved in the progression of cardiopathy. Clin Sci 104: 415-420.

Camandaroba ELP, Campos RF, Magalhães JB, Andrade SG 2001. Clonal structure of Trypanosoma cruzi Colombian strain (biodeme type III): biological, isoenzymic and histopathological analysis of seven isolated clones. Rev Soc Bras Med Trop 34: 151-157.

Camandaroba ELP, Pinheiro LCM, Andrade SG 2002. Oral transmission of Chagas disease: importance of Trypanosoma cruzi biodeme in the intragastric experimental infection. Rev Inst Med Trop Sao Paulo 44: 97-103.

Camargo ME 1966. Fluorescent antibody test for the diagnosis of American trypanosomiasis. Technical modification employing preserved culture forms of Trypanosoma cruzi in a slide test. Rev Inst Med Trop Sao Paulo 8: 227-234.

Campos RMF, Andrade SG 1996. Characterization of subpopulations (clones and subclones) of the 21SF strain of Trypanosoma cruzi after long lasting maintenance in the laboratory. Mem Inst Oswaldo Cruz 91: 795-800.

Coura JR 2013. Chagas disease: control, elimination and eradication. Is it possible? Mem Inst Oswaldo Cruz 108: 962-967.

Dias JCP, Silveira AC, Schofield CJ 2002. The impact of Chagas disease control in Latin America - A Review. Mem Inst Oswaldo Cruz 97: 603-612.

dos Santos DM, Talvani A, Guedes PM, Machado-Coelho GL, de Lana M, Bahia MT 2009. Trypanosoma cruzi: genetic diversity influences the profile of immunoglobulins during experimental infection. Exp Parasitol 121: 8-14.

Federici EE, Abelman WE, Neva FA 1964. Chronic and progressive myocarditis in $\mathrm{C} 3 \mathrm{H}$ mice infected with Trypanosoma cruzi. Am J Trop Med Hyg 3: 272-280.

Lutsiak CME, Semnani RT, Pascalis R, Kashmiri SVS, Schiom J, Sabzevari H 2005. Inhibition of CD4 25 T regulatory cell function implicated in enhanced immune response by low-dose cyclophosphamide. Blood 105: 2862-2868.

Macedo V 1976. Influência da exposição à reinfecção na evolução da doença de Chagas. Rev Patol Trop 5: 33-116.

Murata M, Suzuki T, Midorikawa K, Oikawa S, Kawanishi S 2004. Oxidative DNA damage induced by a hydroperoxide derivative of cyclophosphamide. Free Radic Biol Med 37: 793-802.

Pereira IR, Vilar-Pereira G, da Silva AA, Lannes-Vieira J 2014. Severity of chronic experimental Chagas heart disease parallels tumour necrosis factor and nitric oxide levels in the serum: models of mild and severe disease. Mem Inst Oswaldo Cruz 109: 289-298.

Shevach EM, McHugh RS, Piccirillo CA, Thornton AM 2001. Control of T-cell activation by $\mathrm{CD} 4{ }^{+} \mathrm{CD} 25^{+}$suppessor T cells. Immunol Rev 182: 58-67.

Thé TS, Portella RS, Guerreiro ML, Andrade SG 2013. Effect of treatment with cyclophosphamide in low doses upon the onset of delayed type hypersensitivity in mice chronically infected with Trypanosoma cruzi: involvement of heart interstitial dendritic cells. Mem Inst Oswaldo Cruz 108: 691-698.

Warren LG 1960. Metabolism of Schizotrypanum cruzi Chagas. I. Effect of culture age and substrate concentration on respiratory rate. J Parasitol 46: 529-539. 